\title{
Effect of betalain (betanin) supplementation on endothelial dysfunction and vascular tone in human umbilical vascular endothelial cells
}

\author{
A.K.F. Cheok ${ }^{1}$, A. Rodriguez-Mateos ${ }^{1}$, T.W. George ${ }^{1}$ and P.W. Caton ${ }^{1,2}$ \\ ${ }^{1}$ Department of Nutritional Sciences and ${ }^{2}$ Department of Diabetes, Faculty of Life Science and Medicine, King's \\ College London, SE1 9NH, UK
}

Cardiovascular disease (CVD) has become the leading cause of death in many western-industrialised countries and recent animal studies revealed that supplementation of betalain-rich foods may improve key vascular parameters ${ }^{(1,2)}$. Betalains are bioactive alkaloidal colour pigments that naturally occur in plants like beetroot and dragon fruit ${ }^{(3)}$. Using cell culture models, we aimed to determine the direct cause-and-effect and cardioprotective properties of beetroot-derived betanin (also known as one of the most common food dyes E162).

In this study, we employed two different experimental models namely: preventative and therapeutic models. The former is based on previous work ${ }^{(4)}$ while the latter is a model we developed as a more physiologically possible alternative. In the preventative model, primary HUVECs were pre-treated with betanin $(0 \cdot 5-20 \mu \mathrm{M})$ before challenged with TNF- $\alpha(200 \mathrm{U} / \mathrm{mL})$ to induce endothelial dysfunction followed by a second betanin treatment of the same concentration. In contrast, the therapeutic model does not utilise a pre-treatment phase. In brief, HUVECs were first subjected to TNF- $\alpha(200 \mathrm{U} / \mathrm{mL})$ prior to a shorter treatment duration of betanin $(0 \cdot 5-20 \mu \mathrm{M})$. All experiments were done in quadruplicate and relative gene expression of vascular inflammatory biomarkers (ICAM-1, VCAM-1, and ET-1) were evaluated using real-time reverse-transcriptase quantitative Polymerase Chain Reaction (RT-qPCR).

We observed similar results in both models (Fig. 1a-c \& Fig. 1d-f). Upon stimulation with TNF- $\alpha$, all inflammatory genes were significantly upregulated. Treatment with betanin completely ameliorated this change but we observe no dose-dependent reaction.

(a)

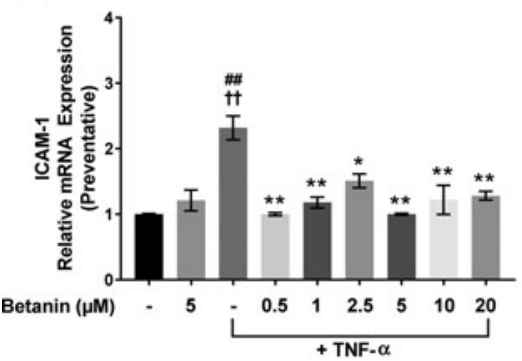

(d)

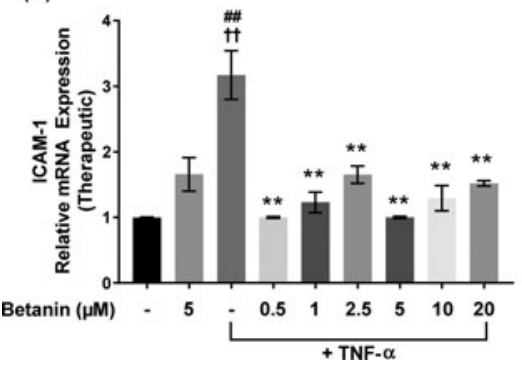

(b)

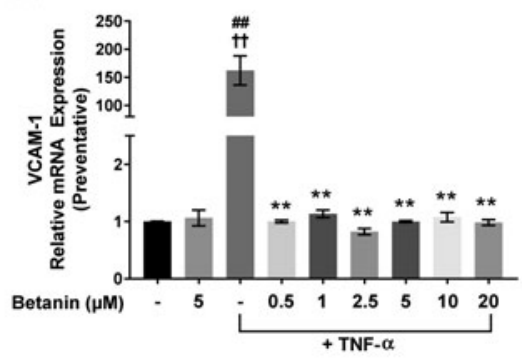

(e)

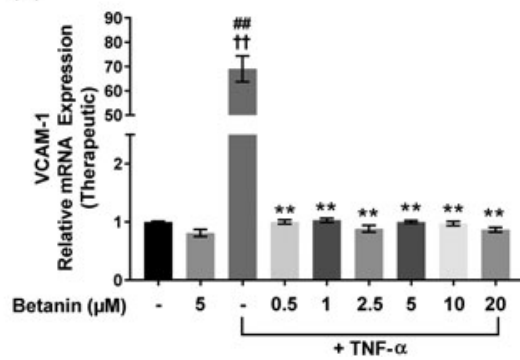

(c)

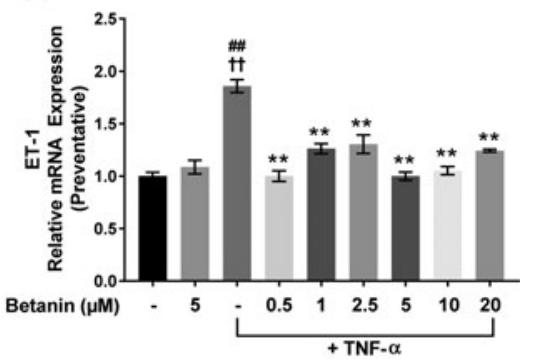

(f)

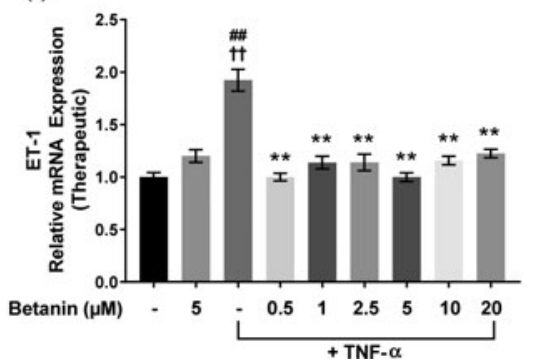

Fig. 1. Preventative model: Relative mRNA expression of (a) ICAM-1, (b) VCAM-1 and (c) ET-1. Therapeutic model: Relative mRNA expression of (d) ICAM-1, (e) VCAM-1 and (f) ET-1. Relative expression is calculated using formula $2^{-(\Delta \Delta \mathrm{Ct})}$ with control $=1$. Data represented as means and SEM of 6 independent experiments $(\mathrm{n}=4)$. ${ }^{\#} \mathrm{p}<0.0001$, vs. control; ${ }^{\dagger} \mathrm{p}<0.0001$, vs. betanin control $(5 \mu \mathrm{M}) ;{ }^{*} \mathrm{p}<0.05,{ }^{* *} \mathrm{p}<0.0001$, vs. TNF- $\alpha$ control (ANOVA, followed by Tukey's test)

Betanin's ability to downregulate ICAM-1 and VCAM-1 confirms its potential as a strong anti-atherogenic agent. Furthermore, it may also promote vasodilatation by suppressing ET-1 expression, consequently lowering blood pressure. Taken together, betanin may offer novel therapeutic options in the treatment and prevention of atherosclerosis and hypertension.

1. Anand Swarup KR, Sattar MA, Abdullah NA et al. (2010) Pharmacognosy Res 2, 31-35.

2. Ramli NS, Brown L, Ismail P et al. (2014) BMC Complem Altern M 14, 189.

3. Khan MI \& Giridhar P (2015) Phytochemistry 117, 267-295.

4. Gentile C, Tesoriere L, Allegra M et al. (2004) Ann NY Acad Sci 1028, 481-486. 